\title{
Gaussian Curvature of Graph-Like Surfaces in 3-Dimensional Hyperbolic Space
}

\author{
Zonglao Zhang \\ College of Mathematics and Information Science, Wenzhou University, Wenzhou 325035, Zhejiang, China \\ E-mail: zonglao@163.com \\ Zhufeng Wang (Corresponding author) \\ College of Continuing Education, Wenzhou University, Wenzhou 325027, Zhejiang, China \\ E-mail: zhufwang@163.com
}

Received: October 8, 2011 Accepted: October 31, 2011 Published: February 1, 2012

doi:10.5539/jmr.v4n1p30 URL: http://dx.doi.org/10.5539/jmr.v4n1p30

\begin{abstract}
In this paper, we investigate the Gaussian curvature of graph-like surfaces in 3-dimensional hyperbolic space. We prove that the graph defined by a radially symmetric function with nonpositive Gaussian curvature is necessarily a surface with Gaussian curvature zero.
\end{abstract}

Keywords: Hyperbolic space, Graph-like surface, Gaussian curvature

\section{Introduction}

The differential geometry of surfaces (or hypersurfaces) in hyperbolic space has been studied by many authors in recent years. Lin (Lin, 1989) and Sa Earp \& Toubiana (Sa Earp \& Toubiana, 2000) investigated the minimal graphs in hyperbolic space. De Silva \& Spruck (De Silva \& Spruck, 2009), Lopez (Lopez, 2001) and Nitsche (Nitsche, 2002) studied more generalized graphs in hyperbolic space with constant or arbitrary mean curvature. Rosenberg \& Spruck (Rosenberg \& Spruck, 1994) and Guan et al. (Guan, Spruck \& Szapiel, 2009; Guan \& Spruck, 2010) investigated the Gaussian curvature and other curvatures of hypersurfaces in hyperbolic space. But still little is known about the Gaussian curvature of surfaces in hyperbolic space. In this paper. we will investigate the Gaussian curvature of graph-like surfaces in 3-dimensional hyperbolic space.

In this paper we use a model of hyperbolic space which was first introduced by Nitsche (Nitsche, 2002). We call this model the Nitsche's model of hyperbolic space. One can also refer to Zhang (Zhang, 2005) for an explanation of the Nitsche's model. Now we give a quick review of this model. Let $\mathbb{H}^{n+1}$ be the upper halfspace model of hyperbolic space with curvature -1 , that is,

$$
\mathbb{H}^{n+1}=\left\{\left(x, x_{n+1}\right) \in \mathbb{R}^{n+1}: x_{n+1}>0\right\}
$$

equipped with the hyperbolic metric

$$
d s^{2}=\frac{\left(d x_{1}\right)^{2}+\left(d x_{2}\right)^{2}+\ldots+\left(d x_{n+1}\right)^{2}}{\left(x_{n+1}\right)^{2}},
$$

where $x=\left(x_{1}, x_{2}, \ldots, x_{n}\right)$. Define mapping

$$
\Psi: D^{n} \times \mathbb{R} \rightarrow \mathbb{H}^{n+1}, \quad \Psi(x, t)=\frac{2 e^{t}}{1+|x|^{2}}\left(x, \frac{1-|x|^{2}}{2}\right),
$$

where $|\cdot|$ denotes the Euclidean norm, and $D^{n} \equiv\left\{x \in \mathbb{R}^{n}:|x|<1\right\}$. Then $\Psi$ is a diffeomorphism. Let $\bar{g}$ be the pull-back metric of the hyperbolic metric $d s^{2}$ of $\mathbb{H}^{n+1}$. Then $D^{n} \times \mathbb{R}$ equipped with metric $\bar{g}$ is the Nitsche's model of hyperbolic space. From now on we denote by $\mathcal{H}^{n+1}$ the Nitsche's model of hyperbolic space.

In this paper we only consider 3-dimensional hyperbolic space $\mathcal{H}^{3}=D^{2} \times \mathbb{R}$. Let $\Omega$ be an open subset of $D^{2}$ and $\varphi$ be a smooth function defined on $\Omega$. Then

$$
\Sigma=\{(x, \varphi(x)) \in \Omega \times \mathbb{R}: x \in \Omega\} \subset \mathcal{H}^{3}
$$

is a surface of $\mathcal{H}^{3}$. We call $\Sigma$ the graph of $\varphi\left(\Sigma\right.$ is also called a graph-like surface of $\left.\mathcal{H}^{3}\right)$. If $\Omega=B_{R}$, the open disc of $\mathbb{R}^{2}$ with center $o$ (the origin of $\mathbb{R}^{2}$ ) and (Euclidean) radius $R<1$, and $\varphi(x)=\Phi(r(x))$ for some function $\Phi(r)(x \in \Omega)$, where 
$r=r(x)$ denotes the hyperbolic distance from $o$ to $x$, then we say that $\varphi$ is radially symmetric (around $o$ ). If $\varphi$ is radially symmetric, the graph $\Sigma$ of $\varphi$ is called a radial graph.

Let $\Omega \subset D^{2}, \varphi \in C^{\infty}(\Omega)$ and $\Sigma$ be the graph of $\varphi$. We recall the definition of Gaussian curvature $K$ of graph $\Sigma$. Let $\bar{\nabla}$ and $\nabla$ be the Riemannian connections of $\mathcal{H}^{3}$ and $\Sigma$, respectively. For any $p \in \Sigma$ and $u, v \in T_{p} \Sigma$ (the tangent space of $\Sigma$ at $p$ ), define

$$
I I(u, v)=\left.\left(\bar{\nabla}_{U} V-\nabla_{U} V\right)\right|_{p},
$$

where $U, V$ are vector fields on $\Sigma$ such that $\left.U\right|_{p}=u,\left.V\right|_{p}=v$. Define $l$ by

$$
I I(u, v)=-l(u, v) \xi,
$$

where $\xi$ denotes a unit normal vector field of $\Sigma$. We call $l$ the real valued second fundamental form of $\Sigma$. The Gaussian curvature $K$ of $\Sigma$ at a point $p \in \Sigma$ is defined by

$$
K(p)=\frac{l(u, u) l(v, v)-l(u, v)^{2}}{\bar{g}(u, u) \bar{g}(v, v)-\bar{g}(u, v)^{2}}
$$

where $u, v \in T_{p} \Sigma$ is linear independent (Gallot et al., 1987).

The main result of this paper is the following:

Main Theorem Let $\Omega=B_{R} \subset D^{2}(0<R<1)$ and $\varphi \in C^{\infty}(\Omega)$. Let $\Sigma=\{(x, \varphi(x)) \mid x \in \Omega\}$ be the graph of $\varphi$ in hyperbolic space $\mathcal{H}^{3}=D^{2} \times \mathbb{R}$ and $K$ be the Gaussian curvature of $\Sigma$. If $\varphi$ is radially symmetric around $o$ and $K \leq 0$, then $\varphi$ is constant on $\Omega$ and $K \equiv 0$.

Remark It is a well-known fact that $D^{n} \times\{t\}$ is a totally geodesic submanifold of hyperbolic space $\mathcal{H}^{n+1}=D^{n} \times \mathbb{R}$ for each $t \in \mathbb{R}$ (Zhang, 2005). So, in the above Main Theorem, the claim that $\varphi$ is constant means that the graph $\Sigma$ defined by $\varphi$ is totally geodesic in $\mathcal{H}^{3}$.

\section{Preliminaries}

Consider the Nitsche's model of 3-dimensional hyperbolic Space $\mathcal{H}^{3}=D^{2} \times \mathbb{R}$. Let $\bar{g}$ be the hyperbolic metric of $\mathcal{H}^{3}$. For every $t \in \mathbb{R}$, it is easy to see that $\left.\bar{g}\right|_{D^{2} \times\{t\}}$ is just the hyperbolic metric of the unit Poincare disk $D^{2}$ :

$$
g=\frac{4|d x|^{2}}{\left(1-|x|^{2}\right)^{2}},
$$

where $|d x|^{2}$ denotes the Euclidean metric on $D^{2}$. Note that

$$
D^{2}=\{z \in \mathbb{C}:|z|<1\} .
$$

Let

$$
z=\rho e^{i \theta}, \quad \rho=\tanh \frac{r}{2},
$$

where $\rho \in[0,1)$ and $r \in[0, \infty) .(r, \theta)$ are the hyperbolic geodesic polar coordinates on $D^{2}$. Then the hyperbolic metric of the unit Poincaré disk $D^{2}$ can be expressed as

$$
g=(d r)^{2}+\sinh ^{2} r d \theta^{2} .
$$

(Chavel, 1984). Hence the hyperbolic metric of $\mathcal{H}^{3}$ can be expressed as

$$
\begin{array}{rlr}
\bar{g} & =\frac{4}{\left(1-|x|^{2}\right)^{2}}|d x|^{2}+\frac{\left(1+|x|^{2}\right)^{2}}{\left(1-|x|^{2}\right)^{2}} d t^{2} & \text { (Nitsche, P.-A., 2002) } \\
& =(d r)^{2}+\sinh ^{2} r d \theta^{2}+\frac{\left(1+|x|^{2}\right)^{2}}{\left(1-|x|^{2}\right)^{2}} d t^{2} \quad \text { (by Eq. (3) and Eq. (4)) } \\
& =(d r)^{2}+\sinh ^{2} r d \theta^{2}+\cosh ^{2} r d t^{2} &
\end{array}
$$

Now we recall some notations about product manifolds (O'Neill, 1983). Consider the product $D^{2} \times \mathbb{R}$. Let

$$
\pi: D^{2} \times \mathbb{R} \rightarrow D^{2}, \quad \pi(x, t)=x,
$$


and

$$
\sigma: D^{2} \times \mathbb{R} \rightarrow \mathbb{R}, \quad \pi(x, t)=t,
$$

be the projections of the product $D^{2} \times \mathbb{R}$. For any $(x, t) \in D^{2} \times \mathbb{R}, \pi^{-1}(x)$ and $\sigma^{-1}(t)$ are called the fibers and the leaves of the product $D^{2} \times \mathbb{R}$, respectively. A vector at a point of $\mathcal{H}^{3}$ tangent to the leaf at this point is called to be horizontal, and tangent to the fiber at this point is called to be vertical. Then functions or vector fields on the factor spaces $\left(D^{2}\right.$ and $\left.\mathbb{R}\right)$ can be viewed, by lifts (horizontal or vertical lifts) via the above projections, as functions or vector fields on $\mathcal{H}^{3}=D^{2} \times \mathbb{R}$. For details see (O'Neill, 1983). That is to say, if $X$ is a vector field on $D^{2}$, then there exists a unique horizontal vector field $X^{*}$ on $\mathcal{H}^{3}=D^{2} \times \mathbb{R}$ such that $d \pi\left(X^{*}\right)=X$ (we call $X^{*}$ the horizontal lift of $X$ ); but, for convenience, we will use the same symbol $X$ to denote both the vector fields $X^{*}$ and $X$ (the exact meaning of $X$ can be known by the context.). In a similar way, for a vector field $U$ on $\mathbb{R}$, we also have a unique vertical vector field $U^{*}$ on $\mathcal{H}^{3}$ such that $d \sigma\left(U^{*}\right)=U$. We also use $U$ to denote both $U$ and $U^{*}$.

\section{Proof of the Main Theorem}

Proof of the Main Theorem: At first we calculate the unit normal vector field $\xi$ of $\Sigma$. Define mapping

$$
\Lambda: \Omega \subset D^{2} \rightarrow \Sigma \subset \mathcal{H}^{3}, \quad x \mapsto(x, \varphi(x)), \quad(x \in \Omega) .
$$

Let $\partial_{r}$ and $\partial_{\theta}$ be the coordinate vector fields of geodesic polar coordinates $(r, \theta)$ on $D^{2}$. Define

$$
v_{1}=d \Lambda\left(\partial_{r}\right), \quad v_{2}=d \Lambda\left(\partial_{\theta}\right) .
$$

A calculation shows that

$$
v_{1}=\partial_{r}+\frac{\partial \varphi}{\partial r} \partial_{t}, \quad v_{2}=\partial_{\theta}+\frac{\partial \varphi}{\partial \theta} \partial_{t},
$$

where $\partial_{t}$ denotes the coordinate vector field of $\mathbb{R}$ (here viewed as a vector field on $\mathcal{H}^{3}$ ). For simplicity, from now on we let $f=\cosh r$. Then one can verifies directly that the unit normal vector field of $\Sigma$ can be chosen as following

$$
\xi=\frac{\partial_{t}-f^{2} \operatorname{grad} \varphi}{\left\|\partial_{t}-f^{2} \operatorname{grad} \varphi\right\|_{\mathcal{H}^{3}}},
$$

where $\operatorname{grad} \varphi$ denotes the gradient of $\varphi$ in the hyperbolic metric of $D^{2}$.

Now we calculate the Christoffel symbols of the Poincare disk $D^{2}$ with respect to the geodesic polar coordinates. For convenience, we rewrite $\partial_{r}=\partial_{1}$ and $\partial_{\theta}=\partial_{2}$. The metric matrix is

$$
\left(\begin{array}{ll}
g_{11} & g_{12} \\
g_{21} & g_{22}
\end{array}\right)=\left(\begin{array}{cc}
1 & 0 \\
0 & \sinh ^{2} r
\end{array}\right)
$$

where $g_{i j}=g\left(\partial_{i}, \partial_{j}\right)$. The inverse of $\left(g_{i j}\right)$ is

$$
\left(\begin{array}{ll}
g^{11} & g^{12} \\
g^{21} & g^{22}
\end{array}\right)=\left(\begin{array}{cc}
1 & 0 \\
0 & \frac{1}{\sinh ^{2} r}
\end{array}\right) .
$$

The Christoffel symbols of the Poincaré disk $D^{2}$ are

$$
\Gamma_{i j}^{k}=\frac{1}{2} \sum_{s} g^{k s}\left(\partial_{j} g_{s i}-\partial_{s} g_{i j}+\partial_{i} g_{j s}\right)=\frac{1}{2} g^{k k}\left(\partial_{j} g_{k i}-\partial_{k} g_{i j}+\partial_{i} g_{j k}\right) .
$$

Then a calculation gives the Christoffel symbols of Poincaré disk $D^{2}$

$$
\left\{\begin{array}{l}
\Gamma_{11}^{1}=\Gamma_{11}^{2}=\Gamma_{22}^{2}=\Gamma_{12}^{1}=0, \\
\Gamma_{22}^{1}=-\sinh r \cosh r, \\
\Gamma_{12}^{2}=\operatorname{coth} r .
\end{array}\right.
$$

Now suppose that $\varphi$ is radially symmetric, let $\varphi(x)=\Phi(r(x))$. We compute the Gaussian curvature $K$ of $\Sigma$. Let $\varphi_{i}=$ $\partial_{i} \varphi, \varphi_{i j}=\partial_{j} \partial_{i} \varphi$. By Eq. (1), Eq. (6) and Eq. (7), the second fundamental form of $\Sigma$ is given by

$$
\begin{aligned}
l\left(v_{i}, v_{j}\right) & =-\bar{g}\left(I I\left(v_{i}, v_{j}\right), \xi\right)=-\bar{g}\left(\bar{\nabla}_{v_{i}} v_{j}-\nabla_{v_{i}} v_{j},, \xi\right)=-\bar{g}\left(\bar{\nabla}_{v_{i}} v_{j}, \xi\right) \\
& =-\frac{1}{\left\|\partial_{t}-f^{2} \operatorname{grad} \varphi\right\|_{\mathcal{H}^{3}}} \bar{g}\left(\bar{\nabla}_{\partial_{i}} \partial_{j}+\varphi_{j} \bar{\nabla}_{\partial_{i}} \partial_{t}+\varphi_{i j} \partial_{t}+\varphi_{i} \bar{\nabla}_{\partial_{t}} \partial_{j}+\varphi_{i} \varphi_{j} \bar{\nabla}_{\partial_{t}} \partial_{t}, \partial_{t}-f^{2} \operatorname{grad} \varphi\right) .
\end{aligned}
$$


Note that $\operatorname{grad} \varphi=\Phi^{\prime} \partial_{r}\left(\right.$ here $\left.\Phi^{\prime}=\frac{d \Phi}{d r}\right)$. By use of Eq. (5), after a tedious computation, we get

$$
\bar{\nabla}_{\partial_{i}} \partial_{t}=\bar{\nabla}_{\partial_{t}} \partial_{i}=\frac{\partial_{i} f}{f} \partial_{t}=\left\{\begin{array}{cl}
\frac{f^{\prime}}{f} \partial_{t}, & \text { if } i=1, \\
0, & \text { if } i=2,
\end{array}\right.
$$

and

$$
\bar{\nabla}_{\partial_{t}} \partial_{t}=-\frac{f^{2}}{f} \operatorname{grad} f=-f f^{\prime} \partial_{r}
$$

Since leaves are totally geodesic, so $\bar{\nabla}_{\partial_{i}} \partial_{j}=D_{\partial_{i}} \partial_{j}$ (here D denotes the covariant derivative with respect to the hyperbolic metric of $D^{2}$ ). Thus from Eq. (9) we get

$$
\left\{\begin{array}{l}
\bar{\nabla}_{\partial_{1}} \partial_{1}=D_{\partial_{1}} \partial_{1}=\Gamma_{11}^{1} \partial_{1}+\Gamma_{11}^{2} \partial_{2}=0 \\
\bar{\nabla}_{\partial_{2}} \partial_{2}=D_{\partial_{2}} \partial_{2}=\Gamma_{22}^{1} \partial_{1}+\Gamma_{22}^{2} \partial_{2}=-\sinh r \cosh r \partial_{1}=-f f^{\prime} \partial_{r} \\
\bar{\nabla}_{\partial_{1}} \partial_{2}=D_{\partial_{1}} \partial_{2}=\Gamma_{12}^{1} \partial_{1}+\Gamma_{12}^{2} \partial_{2}=\operatorname{coth} r \partial_{\theta}
\end{array}\right.
$$

Let

$$
C_{0}=\frac{1}{\left\|\partial_{t}-f^{2} \operatorname{grad} \varphi\right\|_{\mathcal{H}^{3}}} .
$$

Thus we have

$$
\begin{aligned}
l\left(v_{1}, v_{1}\right) & =-C_{0} \bar{g}\left(\bar{\nabla}_{\partial_{1}} \partial_{1}+\varphi_{1} \bar{\nabla}_{\partial_{1}} \partial_{t}+\varphi_{11} \partial_{t}+\varphi_{1} \bar{\nabla}_{\partial_{t}} \partial_{1}+\left(\varphi_{1}\right)^{2} \bar{\nabla}_{\partial_{t}} \partial_{t}, \partial_{t}-f^{2} \operatorname{grad} \varphi\right) . \\
& =-C_{0} f\left\{\Phi^{\prime \prime} f+2 \Phi^{\prime} f^{\prime}+\left(\Phi^{\prime}\right)^{3} f^{2} f^{\prime}\right\}, \\
l\left(v_{2}, v_{2}\right) & =-C_{0} \bar{g}\left(\bar{\nabla}_{\partial_{2}} \partial_{2}+\varphi_{2} \bar{\nabla}_{\partial_{2}} \partial_{t}+\varphi_{22} \partial_{t}+\varphi_{2} \bar{\nabla}_{\partial_{t}} \partial_{2}+\left(\varphi_{2}\right)^{2} \bar{\nabla}_{\partial_{t}} \partial_{t}, \partial_{t}-f^{2} \operatorname{grad} \varphi\right) \\
& =-C_{0} \Phi^{\prime} f^{3} f^{\prime},
\end{aligned}
$$

and

$$
\begin{aligned}
l\left(v_{1}, v_{2}\right) & =-C_{0} \bar{g}\left(\bar{\nabla}_{\partial_{1}} \partial_{2}+\varphi_{2} \bar{\nabla}_{\partial_{1}} \partial_{t}+\varphi_{12} \partial_{t}+\varphi_{1} \bar{\nabla}_{\partial_{t}} \partial_{2}+\varphi_{1} \varphi_{2} \bar{\nabla}_{\partial_{t}} \partial_{t}, \partial_{t}-f^{2} \operatorname{grad} \varphi\right) . \\
& =0 .
\end{aligned}
$$

Hence

$$
\begin{aligned}
l\left(v_{1}, v_{1}\right) l\left(v_{2}, v_{2}\right)-l\left(v_{1}, v_{2}\right)^{2} & =C_{0}^{2} f\left[\Phi^{\prime \prime} f+2 \Phi^{\prime} f^{\prime}+\left(\Phi^{\prime}\right)^{3} f^{2} f^{\prime}\right] \Phi^{\prime} f^{3} f^{\prime} \\
& =C_{0}^{2} f^{4} f^{\prime}\left[\Phi^{\prime} \Phi^{\prime \prime} f+2\left(\Phi^{\prime}\right)^{2} f^{\prime}+\left(\Phi^{\prime}\right)^{4} f^{2} f^{\prime}\right]
\end{aligned}
$$

We know that, at every point $p$ of $\Sigma(p \neq \Lambda(o))$,

$$
\bar{g}\left(v_{1}, v_{1}\right) \bar{g}\left(v_{2}, v_{2}\right)-\bar{g}\left(v_{1}, v_{2}\right)^{2}>0 .
$$

So by Eq. (2) and from the hypothesis of the theorem that $K \leq 0$, we get

$$
l\left(v_{1}, v_{1}\right) l\left(v_{2}, v_{2}\right)-l\left(v_{1}, v_{2}\right)^{2} \leq 0
$$

at $p \neq \Lambda(o)$. Then Eq. (17) yields, for any $0<r<R_{h}\left(R_{h}\right.$ denotes the hyperbolic radius of $\Omega$ ) (and hence for any $\left.0 \leq r<R_{h}\right)$,

$$
\Phi^{\prime} \Phi^{\prime \prime} f+2\left(\Phi^{\prime}\right)^{2} f^{\prime}+\left(\Phi^{\prime}\right)^{4} f^{2} f^{\prime} \leq 0 .
$$

Therefore,

$$
\Phi^{\prime} \Phi^{\prime \prime} \leq 0 .
$$

The radial symmetry of $\varphi$ implies that $\Phi^{\prime}(0)=0$. So we have

$$
\frac{\left[\left(\Phi^{\prime}\right)^{2}\right]^{\prime}}{2}=\Phi^{\prime} \Phi^{\prime \prime} \leq 0
$$


on $\left[0, R_{h}\right)$. Now $\left(\Phi^{\prime}\right)^{2}$ is decreasing on $\left[0, R_{h}\right)$. But $\Phi^{\prime}(0)=0$ and $\left(\Phi^{\prime}\right)^{2} \geq 0$, it follows that $\Phi^{\prime}(r) \equiv 0$ and hence $\Phi(r)$ is constant on $\left[0, R_{h}\right)$, and hence $\varphi$ is constant on $\Omega$. From Eq. (17) we also know $K \equiv 0$. The proof of the main theorem is finished.

\section{References}

Chavel, I. (1984). Eigenvalues in Riemannian geometry, New York: Academic Press.

De Silva, D., \& Spruck, J. (2009). Rearrangements and radial graphs of constant mean curvature in hyperbolic space. Calc. Var. Partial Differential Equations, 34, 73-95. http://dx.doi.org/10.1007/s00526-008-0176-3

Gallot, S., Hulin, D., \& Lafontaine, J. (1987). Riemannian geometry, Berlin: Springer-Verlag. (Chapter V).

Guan, B., \& Spruck, J. (2010). Hypersurfaces of constant curvature in hyperbolic space II. J. Eur. Math. Soc., 12, 797-817. http://dx.doi.org/10.4171/JEMS/215

Guan, B., Spruck, J., \& Szapiel, M. (2009). Hypersurfaces of constant curvature in hyperbolic space I. J. Geom. Anal., 19, 772-795. http://dx.doi.org/10.1007/s12220-009-9086-7

Lin, F. H. (1989). On the Dirichlet problem for minimal graphs in hyperbolic space. Invent. math., 96, 593-612.

Lopez, R. (2001). Graphs of constant mean curvature in hyperbolic space. Ann. Global Anal. Geom., $20,59-75$. http://dx.doi.org/10.1023/A:1010676217144

Nitsche, P.-A. (2002). Existence of prescribed mean curvature graphs in hyperbolic space. Manuscripta Math., 108, 349-367. http://dx.doi.org/10.1007/s002290200267

O’Neill, B. (1983). Semi-Riemannian geometry with applications to relativity, London:Academic Press.

Rosenberg, H., \& Spruck, J. (1994). On the existence of convex hypersurfaces of constant Gauss curvature in hyperbolic space. J. Differential Geom., 40, 379-409.

Sa Earp R., \& Toubiana E. (2000). Existence and uniqueness of minimal graphs in hyperbolic space. Asian J. Math., 4, 669-694.

Zhang, Zonglao. (2005). A remark on the mean curvature of a graph-like hypersurface in hyperbolic space. J. Math. Anal. Appl., 305, 491-501. http://dx.doi.org/10.1016/j.jmaa.2004.11.032 